Editorial

Z Gerontol Geriat 2019·52 (Suppl 3):S165-S167 https://doi.org/10.1007/s00391-019-01597-1

(C) Springer Medizin Verlag GmbH, ein Teil von Springer Nature 2019
Heiner Fangerau ${ }^{1}$ Andrea von Hülsen-Esch ${ }^{2}$ Daniel Schäfer ${ }^{3}$

${ }^{1}$ Institut für Geschichte, Theorie und Ethik der Medizin, Heinrich-Heine-Universität Düsseldorf, Medizinische Fakultät, Düsseldorf, Deutschland

${ }^{2}$ Institut für Kunstgeschichte, Heinrich-Heine-Universität Düsseldorf, Düsseldorf, Deutschland

${ }^{3}$ Institut für Geschichte und Ethik der Medizin, Uniklinikum Köln, Universität zu Köln, Köln, Deutschland

\title{
Kulturelle Aspekte des Alter(n)s - die Wirkung von Altersstereotypien auf die Alltagspraxis
}

Eine bestimmte Haltung dem Alter und alten Menschen gegenüber fließt oftmals als nichtbewusste Grundannahme in das Regelwerk des gesamtgesellschaftlichen Gefüges ein. Dieser Umgang mit dem Alter ist wiederum geprägt von der Kultur - Kultur aber ist ein schillernder Begriff. Nähert man sich ihm etymologisch, so zeigt er schon in der Antike zwei Schattierungen. Auf der einen Seite wird er der Natur gegenübergestellt, wenn mit ihm die von Menschen geleistete Bearbeitung der Natur gemeint ist. Auf der anderen Seite wird er weiter gefasst und bezeichnet auch die Arbeit des Geistes und die an der eigenen Persönlichkeit. Diese Doppelbödigkeit aufnehmend und weiter konkretisierend wird heute oft ein ergologischer Kulturbegriff, der das menschliche Wirken auf die Natur bezeichnet, von einem interpretativen Kulturbegriff unterschieden, der die symbolischen Produktionen und die jeweilige Interpretation ihrer Bedeutung in den Mittelpunkt rückt. Mit einem solchen Kulturverständnis verschwimmt die vordergründig so klare Grenze zwischen Natur und Kultur ebenso wie die zwischen den Naturund Kultur-/Geisteswissenschaften [18]. Auch die Frage des Alters wird so zu einem nicht allein von einer Seite aus $\mathrm{zu}$ analysierenden und erforschenden Problem. Alter erhält eine Doppelgesichtigkeit. Neben biologisch ermittelbaren Alterungsprozessen, die schon mit der Geburt beginnen, ist die Fra- ge der jeweiligen Interpretation eines Zustands im Sinne einer Einordnung in Symbol- und Deutungswelten einer Gesellschaft bedeutsam. Kultur ist keine unveränderliche Setzung, sondern wandelt sich in ihrer raumzeitlichen Vielfalt und wirkt auf diese Vielfalt zurück. Insbesondere die kulturwissenschaftliche Forschung macht spätestens seit der Jahrtausendwende mit verschiedenen Ansätzen deutlich, dass Alter(n) als Gegenstand des Wissens und als Konzept begriffen werden muss, und dass Konzeptionen vom Alter(n) ein möglicher Grundbestandteil des Bildes vom Menschen überhaupt sind [4-13, 15, 17, 21-23].

Die Untersuchung kultureller Aspekte des Alters zielt dabei auf den von Menschen erzeugten Gesamtkomplex aus Vorstellungen, Denkformen, Empfindungsweisen, Werten und Bedeutungen, der sich in Symbolsystemen materialisiert. Einer solchen Begriffsbestimmung zufolge sind nicht nur materiale (z.B. künstlerische) Ausdrucksformen zum Bereich der Kultur zu zählen, sondern auch die sozialen Institutionen und mentalen Dispositionen, die die Hervorbringung solcher Artefakte überhaupt erst ermöglichen. So verstanden ist Kultur „Formel und Praxis einer gesellschaftlichen Selbstverständigung “ [1, S. 9f.] und somit das umfassende Merkmal für eine gesellschaftliche Prägung: von der Sprache und der historischen Tradition über die literarischen und medialen Äu-
Berungsformen, die strukturgebenden Formen des Zusammenlebens und der sozialen Gruppenbildung bis hin zum Handeln in wirtschaftlichen Unternehmen. Die Gesellschaft ist ein Teil von Kultur, Bestandteil der immateriellen Kultur, die den Beziehungen zwischen Menschen Struktur verleiht [19, S. 46]. Bezogen auf kulturelle Aspekte des Alters ist somit u.a. zu fragen:

- nach dem kulturellen Gedächtnis,

- nach der Verstetigung kultureller

Ordnungsmuster und Normen,

- nach Altersstereotypien, die sich herausbilden,

- nach der Entstehung und Wirkung von Sinnformationen,

- nach dem spezifischen Profil der europäischen Kultur im Vergleich zu anderen Kulturen,

- nach Werten und Wertewandel,

- nach kognitiven und emotionalen Dispositionen („Mentalität“),

- nach Ritualen in bestimmten Lebenssituationen und Altersstufen,

- danach, welche Vorstellungen und welche Bilder der Wirklichkeit handlungsleitend sind und sich in Kunst und Literatur äußern [20].

Es geht darum, das Alter(n) selbst als einen kulturellen Prozess zu begreifen, auch um starre Altersstereotypien, die beispielsweise sprachlich intergenerationell weitergegeben werden, bewusst zu machen und aufzubrechen [14, hier S. 399, „Alterslast“, „Überalterung“, 
„Pflegelawine“]. So hat beispielsweise die zunehmend positive Bewertung der individualisierten, aktiven und präventiven Lebensstile $\mathrm{zu}$ einer Abwertung des Alter(n)s beigetragen, mit der Folge, dass bei den im historischen Verlauf ausgeprägten Stereotypen „Weisheit des Alters" auf der einen Seite und „Verfall des Körpers" auf der anderen Seite mehr und mehr die Verfallserscheinungen mit dem Alter verbunden werden [16]. Die Normierung der Lebensphasen über Körpervorstellungen lässt Altersstereotype lebendig werden, die nur dadurch, dass sie ins Bewusstsein gerückt und erkannt, auch überwunden werden können [12]. Die Entwicklung von neuen Konzepten für das Alter(n) und deren Umsetzung in die Praxis werden eine Auseinandersetzung mit diesen Stereotypen nach sich ziehen, für Diversität in der Gesellschaft sensibilisieren, die Sinne für verstellende mediale Praktiken schärfen, den Wert altersbedingter Erfahrung steigern und ein Bewusstsein für das Recht auf kulturelle Teilhabe auch im Alter schaffen. Hier setzen die vernetzten kulturwissenschaftlichen Forschungen an (für einen Forschungsüberblick: [2, 17, 24]), die, ergänzt um natur-, medizin-, rechts- und wirtschaftswissenschaftliche Fragestellungen, weitgespannte Diskurse zusammenführen und künftig mit der Entwicklung von Konzepten in die Praxis vermitteln könnten.

In diesem Sonderheft führen wir drei Blickweisen auf kulturelle Aspekte des Alters in diesem Sinne zusammen und versuchen dabei, interdisziplinäre Spezifika des Blicks auf die Kultur zu illustrieren:

Der Beitrag Schäfer, von Hülsen-Esch und Fangerau bietet eine Analyse kultureller Haupt- oder Nebenbedeutungen von historischen und aktuellen Assistenzsystemen zur Fortbewegung für alte Menschen (insbesondere Stock, Rollstuhl und Rollator) und führt zur These, dass die semantische Vielfalt dieser Systeme in engem Bezug zu den jeweiligen Altersrollen und -stereotypien steht, die in bestimmten Epochen vorherrschend waren. Die hier genutzte Methode verbindet qualitative und komparative Analysen philologisch-medizinhistorischer, kunsthistorischer und technikethischer Quellen.

Der Beitrag Ibrahim und Bayen untersucht die Nähe positiver und negativer Altersstereotypen sowie -attitüden zur jeweiligen „Kultur“ (inkl. deren religiöse Einflussfaktoren). Diese Nähe wird u.a. deutlich in kulturvergleichenden Studien, die allerdings bislang (zu) wenig empirisch gestützt und soziodemografisch differenziert sind und außerdem grundlegende alternative (d.h. möglicherweise nichtkulturelle) Einflussmöglichkeiten noch nicht in Erwägung ziehen: Hierzu gehören der Einfluss des Modernisierungs- und Urbanisierungsgrads einer Gesellschaft und der Einfluss der Geschwindigkeit des Alterungsprozesses sowie der davon beeinflussten ökonomischen Ressourcen einer Gesellschaft. Methodisch basiert der Beitrag auf einer Literaturrecherche und -auswertung internationaler kulturvergleichender Studien vornehmlich aus dem arabischen Sprachraum.

Der Beitrag Weber, Angerer und Müller widmet sich der Analyse signifikanter Zusammenhänge zwischen negativen (Meta-)Altersstereotypen und einem organisationspsychologischen Altersklima auf der einen Seite und verminderter Selbstwirksamkeit, Arbeitszufriedenheit, Leistung, Arbeitsengagement, Lern- oder Entwicklungsbereitschaft sowie gesteigerten Renteneintrittsabsichten älterer Beschäftigter auf der anderen Seite. Als Methode kommt hier ein LiteraturSurvey/-Review (24 internationale relevante Studien, allerdings überwiegend unzureichender Qualität) zum Einsatz.

Wir hoffen, durch diesen hier im Heft geleisteten dreifachen Perspektivenwechsel zeigen zu können, dass es gelingen kann, den Umgang mit dem Alter, statt mit den üblichen biomedizinischen und demografischen Kennziffern zu messen, nicht zuletzt durch die Aufdeckung wirksamer Altersstereotypien mit anderen Kategorien zu bewerten und am Ende Maßnahmen vorzuschlagen, die eine stärkere Teilhabe der alten und hochaltrigen Menschen am Lebensalltag zur Folge haben. Erste unter diesen Gesichtspunkten in die Praxis wirkende Ansätze bieten etwa Angebote zur Verbesserung der Kulturkompetenz im Hinblick auf eine stärkere kulturelle Teilhabe älterer Menschen, wie sie beispielsweise die Akademie Remscheid mit ihrem „Kompetenzzentrum für Kultur und Bildung im Alter" (https://ibkkubia.de/) vermittelt. Es geht darum, das Alter(n) selbst als einen kulturellen Prozess zu begreifen, starre, beschäftigungsbezogene Altersgrenzen mit neuen Beschäftigungsmodellen zu überwinden und ein aktiv gestaltetes Alter(n) einem „produktiven“" [3, S. 127-163, 187-190, 278-285] bzw. einem „erfolgreichen“ [25] Alter(n) entgegenzusetzen.

\section{Korrespondenzadresse}

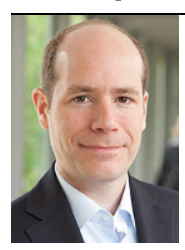

Prof. Dr. Heiner Fangerau

Institut für Geschichte, Theorie und Ethik der Medizin, Heinrich-Heine-

Universität Düsseldorf, Medizinische Fakultät Universitätsstraße 1, 40225 Düsseldorf, Deutschland heiner.fangerau@hhu.de

Prof. Dr. Andrea von
Hülsen-Esch
Institut für Kunstgeschichte,
Heinrich-Heine-Universität
Düsseldorf
Universitätsstr. 1, Geb. 23.32,
40225 Düsseldorf,
Deutschland
huelsen-esch@phil.hhu.de

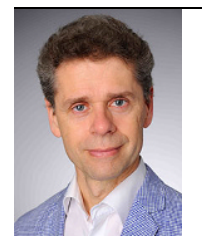

Prof. Dr. med. Dr. phil. Daniel Schäfer Institut für Geschichte und Ethik der Medizin, Uniklinikum Köln, Universität zu Köln

Joseph-Stelzmann-Str. 20, Geb. 42, 50931 Köln,

Deutschland

daniel.schaefer@uni-koeln.de

Interessenkonflikt. H. Fangerau, A. von Hülsen-Esch und D. Schäfer geben an, dass kein Interessenkonflikt besteht.

\section{Literatur}

1. BaeckerD (2000) Wozu Kultur? Kadmos, Berlin

2. Cole TR, Ray RE, Kastenbaum R (Hrsg) (2010) A guide to humanistic studies in aging. What does it mean to grow old. Johns Hopkins University Press, Baltimore

3. Denninger T, van Dyck S, Lessenich S et al (2014) Leben im Ruhestand. Zur Neuverhandlung des Alters in der Aktivgesellschaft. transcript, Bielefeld 
4. Elm D, Fitzon T, Liess $\mathrm{K}$ et al (Hrsg) (2009) Alterstopoi. Das Wissen von den Lebensaltern in Literatur, Kunst und Theologie. de Gruyter, Berlin, New York

5. Fangerau $\mathrm{H}$, Gomille $\mathrm{M}$, Herwig $\mathrm{H}$ et al (Hrsg) (2007) Alterskulturen und Potentiale des Alter(n)s. Akademie-Verlag, Berlin

6. Ferring $D$, Haller M, Meyer-Wolters $\mathrm{H}$ (Hrsg) (2008) Sozio-kulturelle Konstruktionen des Alters. Transdisziplinäre Perspektiven. Königshausen \& Neumann, Würzburg

7. Freiburg R, Kretschmar D (Hrsg) (2012) Alter(n) in Literatur und Kultur der Gegenwart. Königshausen \& Neumann, Würzburg

8. Göckenjan G(2000) Das Alterwürdigen. Suhrkamp, Frankfurta.M.

9. Haller M, Hautzel H (Hrsg) (2009) Lebenslanges Lesen. Lektüreautobiographien, Reflexionen und Analysen. Kisch, Nümbrecht

10. Hartung A (Hrsg) (2011) Lieben und Altern. Die Konstitution von Alter(n)swirklichkeiten im Film. kopaed, München

11. Herrmann-Otto E, Wöhrle G, Harth R (Hrsg) (2004) Die Kultur des Alterns von der Antike bis zur Gegenwart. Röhrig, St. Ingbert

12. Herwig $\mathrm{H}$ (Hrsg) (2009) Alterskonzepte in Literatur, bildender Kunst, Film und Medizin. Rombach, Freiburg

13. Herwig H (Hrsg) (2014) Merkwürdige Alte. Zu einer literarischen und bildlichen Kultur des Alter(n)s. transcript, Bielefeld

14. Hoppe B, Wulf C (1997) Alter. In: Wulf C (Hrsg) Vom Menschen. Handbuch Historische Anthropologie. Beltz, Weinheim, S398-403

15. Hülsen-Esch A (Hrsg) (2015) Alter(n) neu denken. Konzepte für eine neue Alter(n)skultur. transcript Bielefeld

16. Hülsen-Esch A (2009) Falten, Sehnen, Knochen.Zur Materialisierung des Alters in der Kunst um 1500. In: Herwig H (Hrsg) Alterskonzepte in Literatur, bildender Kunst, Film und Medizin. Rombach, Freiburg, S13-44

17. Hülsen-Esch A, Seidler M, Tagsold C (Hrsg) (2013) Methoden der Alter(n)sforschung. Disziplinäre Positionen und transdisziplinäre Perspektiven. transcript, Bielefeld

18. Koschorke A (2009) Zur Epistemologie der Natur/ Kultur-Grenze und zu ihren disziplinären Folgen. Dtsch Vierteljahrsschr Literaturwiss Geistesgesch 83:9-25

19. Oexle OG (2011) Historische Kulturwissenschaft heute. In: Oexle OG (Hrsg) Die Wirklichkeit und das Wissen. Mittelalterforschung - Historische Kulturwissenschaft - Geschichte und Theorie der historischen Erkenntnis. Vandenhoeck \& Ruprecht, Göttingen, S33-58

20. Posner R (2008) Kultursemiotik. In: Nünning A Nünning V (Hrsg) Einführung in die Kulturwissenschaften: Theoretische Grundlagen - Ansätze Perspektiven. Metzler, Stuttgart, S39-72

21. Schäfer D (2004) Alter und Krankheit in der Frühen Neuzeit. Campus, Frankfurt a. M.

22. Thane $P$ (2005) Das Alter. Eine Kulturgeschichte. Wissenschaftliche Buchgesellschaft, Darmstadt

23. Vavra E (Hrsg) (2008) Alterskulturen des Mittelalters und der frühen Neuzeit. Öster. Akad. Wiss., Wien

24. Wahl H-W, Kruse A (Hrsg) (2014) Lebensläufe im Wandel. Entwicklung über die Lebensspanne aus Sicht verschiedener Disziplinen. Kohlhammer, Stuttgart

25. Zimmermann H-P (2012) Dimensionen anderen Alterns: Differenzialität - Othering - Alterität. Medien \& Altern.ZForsch Prax 1:22-36 